\title{
Automatic Compartment Modelling and Segmentation for Dynamical Renal Scintigraphies
}

\author{
Daniel Ståhl ${ }^{1,2}$, Kalle Åström ${ }^{1}$, Niels Christian Overgaard ${ }^{1}$, \\ Matilda Landgren ${ }^{1,2}$, Karl Sjöstrand ${ }^{2,3}$, and Lars Edenbrandt ${ }^{2}$ \\ ${ }^{1}$ Centre for Mathematical Sciences, Lund University, Lund, Sweden \\ \{kalle,nco\}@maths.1th.se \\ ${ }^{2}$ Exini Diagnostics AB, Lund, Sweden \\ \{karl.sjostrand, lars.edenbrandt\}@exini.com \\ ${ }^{3}$ Department of Informatics and Mathematical Modelling, \\ Technical University of Denmark, Kgs. Lyngby, Denmark
}

\begin{abstract}
Time-resolved medical data has important applications in a large variety of medical applications. In this paper we study automatic analysis of dynamical renal scintigraphies. The traditional analysis pipeline for dynamical renal scintigraphies is to use manual or semiautomatic methods for segmentation of pixels into physical compartments, extract their corresponding time-activity curves and then compute the parameters that are relevant for medical assessment. In this paper we present a fully automatic system that incorporates spatial smoothing constraints, compartment modelling and positivity constraints to produce an interpretation of the full time-resolved data. The method has been tested on renal dynamical scintigraphies with promising results. It is shown that the method indeed produces more compact representations, while keeping the residual of fit low. The parameters of the time activity curve, such as peak-time and time for half activity from peak, are compared between the previous semiautomatic method and the method presented in this paper. It is also shown how to obtain new and clinically relevant features using our novel system.
\end{abstract}

Keywords: Medical image analysis, time-resolved, compartment modelling, dynamical renal scintigraphies, segmentation.

\section{Introduction}

Dynamical renal scintigraphy, or simply Renography, is a method used by medical doctors to assess the renal function of a patient. It exploits the mechanisms of the homeostasis - preservation of an optimal extracellular fluid volume and the ability to either remove or restore ions and chemical compounds produced as a result of the metabolism. Since these abilities heavily rely on the complex structure of the kidney any impairments, such as kidney stones, cancer or obstructions, on the kidneys' anatomy and physiology lead to decreased renal function. If this is suspected by the medical doctor, then renography is performed. The procedure of the examination is that the patient is excessively hydrated to build up the

A. Heyden and F. Kahl (Eds.): SCIA 2011, LNCS 6688, pp. 557-568, 2011.

(C) Springer-Verlag Berlin Heidelberg 2011 
urine production. The patient is then given an injection of a tracer consisting of a radioactive isotope, ${ }^{99 m} \mathrm{Tc}$, that is attached to a molecule specifically designed to be removed by the kidneys. The progression of this tracer is then recorded by a gamma camera to form a time-resolved image sequence that is used in the further analysis.

The acquired sequence holds the accumulated counts between time points $t-$ $\Delta t$ and $t$ for each detector on location $(x, y)$ in the frame corresponding to time $t$. The data that is used in this paper was retrieved from Skånes universitetssjukhus, SUS, where they record this progression with a 128-by-128 detector grid and with uniform time sampling, $\Delta t=15$. This provides a resulting image sequence which consists of $n_{p}=80$ frames. In Figure 1 a summarized image sequence is displayed.
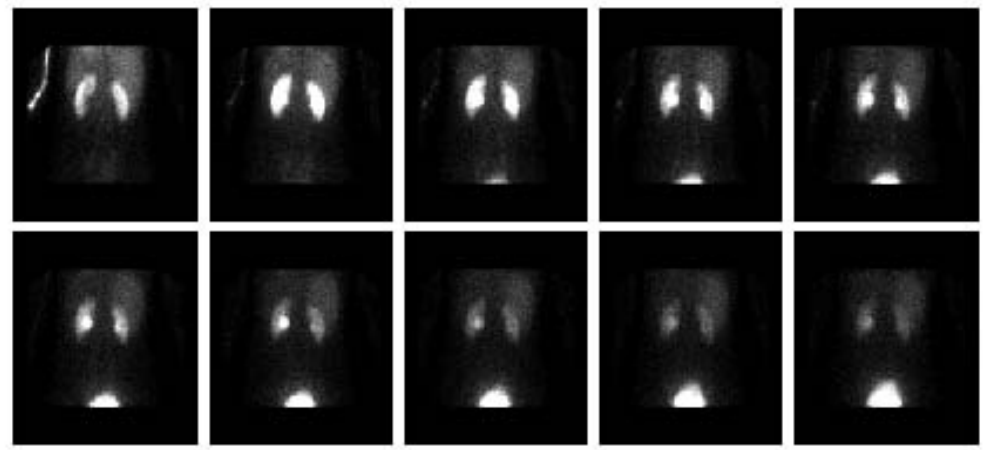

Fig. 1. A summarized image sequence from dynamical renal scintigraphies, the images show the accumulated counts every second minute

The traditional analysis pipeline then is to use manual and/or semi-automatic methods for segmenting the data into pixels, which correspond to the different physical compartments, to estimate the corresponding time-activity curves for the different compartments by summing the pixels in each compartment and to estimate parameters or make classification based on such curves. Two problems arise from the above, (a) manual methods are often time-consuming and different operators may obtain slightly different results and (b) each pixel contains an unknown mixture from the different compartments which is interfering the results. Especially interesting is the way to obtain the Renogram, the TAC which describes the uptake and washout from the kidney and is the primary result of renography studies. In a publication from 2010 Piepsz et al. [2] states "The way of handling a renogram remains extremely variable from country to country and even from department to department. Part of these divergences is obviously related to the use of obsolete software available on most of the gamma camera systems". The result of the previously mentioned problems is the huge variety of renal software packages currently used by practitioners, where many of them 
are developed to fit local fashion e.g. 3, or the one found at SUS. There also exists software from commercial actors trying to bridge the gap 4]. Common for these applications is their manual or semi-automatic approach to obtain the parameters [5].

The aim of this paper is thus to address the problems stated above, (a) and (b), and construct an automatic method to assess the renal function by exploiting the fact that each pixel contains a mixture of contributions from a few types of tissue and organs, in this paper denoted as compartments, which can be identified and used to obtain the structure of tissue and organs that is present in the image sequence. This corresponds to finding the compartments, their time-activity curves and the composition of compartments in each pixel. The result includes data about (i) for each pixel $(\mathrm{x}, \mathrm{y})$, the weight $c_{k}(x, y)$ representing the contribution from each compartment $k$ in the pixel, (ii) the time-dependence $b_{k}(t)$ of each compartment and (iii) system identification of the underlying compartment model. We will do this in the setting of positivity and spatial smoothness constraints applied on $c_{k}$ and $b_{k}$. With this information an analysis of the renal function is performed.

Many articles have been published on how to obtain the image sequence, the renogram and which parameters that can be used for the medical assessment. One way of exploiting information in the data is to use Proper Orthogonal Decomposition, POD, where it was shown by Veltri et al. 11 that a few modes generated from it were sufficient to distinguish different parts of the anatomy of the healthy kidney as well as pathological areas in the pathological kidney. At ISCORN 12010 Richard Lawson presented a summary of the methodologies used when performing an analysis of the kidney and the extraction of the renogram [6]. In his presentation he primarily focused on two strategies for counteracting the accumulated counts from background tissue - the Patlak plot and the deconvolution analysis. Whereas the mathematical model behind the Patlak plot relies on the assumption of constant infusion of tracer, deconvolution analysis models the kidneys' impulse response function from an injection from the blood, cf. [78. Other approaches to extract the renogram are also introduced in [95]. Compartmental modelling has also drawn some attention, in the mid-nineties Fine et al. developed a model for parametric deconvolution analysis [10]. Other attempts include the use of concepts from pharmacokinetics e.g. Meng et al. propose a 2-compartmental model approach to reconstruct the renogram and estimate physical parameters [11. Drainage and flow rate parameters between different compartments can also be estimated using compartment models [12.

\section{Methods}

Our proposed system is built up by several modules further explained in this section. At first we present a brief introduction to the Singular Value Decomposition,

${ }^{1}$ XIV International Symposium on Radionuclides in Nephrourology. 
SVD, which is used as a reference method. Later in this section our method for estimating the weights $c_{k}(x, y)$, the bases $b_{k}(t)$ and the compartmental models are introduced.

Our assumption is that the data $D(x, y, t)$ can be approximated well as a linear combination of these components according to

$$
D(x, y, t) \approx \tilde{D}_{c, b}(x, y, t)=\sum_{k=1}^{K} c_{k}(x, y) b_{k}(t)
$$

where $K$ is the number of compartments, in our experiments $K=5$, and c and b denotes the sets of weights and bases that is reconstructing $D$. Since the image sequence is a recording of the accumulated counts it is further assumed that each element in $D$ can be regarded as generated from a counting process e.g. Poisson process with intensity $\lambda$. However, if the accumulated counts are large it could as well be approximated by a normal distribution. It should also be outlined that scattering and attenuation effects introduces noise in each element of $D$.

\subsection{Using SVD to Approximate the Data}

A common approach to reduce the dimensionality of the data and its noise is to use SVD. The data sequence can then be optimally reconstructed in a least squares sense [13, i.e. the approximation $\tilde{D}_{c, b}$ that minimizes

$$
\min _{\mathbf{c}, \mathbf{b}} \sum_{x} \sum_{y} \sum_{t}\left|D(x, y, t)-\tilde{D}_{c, b}(x, y, t)\right|^{2}
$$

is found by (i) rearranging the data $D$ in a matrix $M$ so that each time point $t$ in $D$ forms a column in $M$ and (ii) performing a singular value decomposition of $M, M=U S V^{T}$. In the case of 5 compartments and 5 basis functions, the first 5 columns of $V$ gives the optimal basis functions and the first 5 columns of $U S$ gives the optimal weights $c_{k}$ after rearrangement of the columns back to matrix form again. An illustration of the output is shown in Figure 2 ,
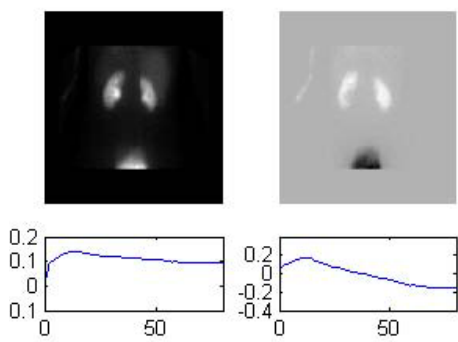
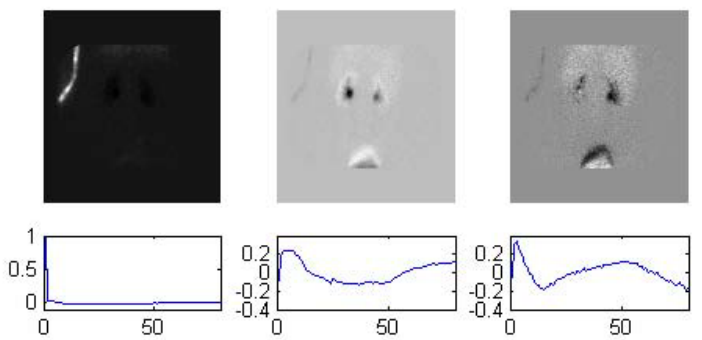

Fig. 2. The first five modes generated by SVD from left to right. The lower row holds the bases and the upper row holds the scores. 
SVD provides the optimal $L^{2}$ fit, but ignores constraints such as sparsity, spatial continuity and positivity. The weights $c_{k}(x, y)$ is also expected to assume both positive and negative parts where most of them will be nonzero. Although no particular spatial continuity constraints are assumed, the weights might still be relatively smooth due to the structure of the data. Note also that the generated bases $b_{k}$ does not in general provide any physiological information about compartment $k$ due to the orthogonality of the bases and that the concentration of tracer only can assume positive values. Hence, the bases are not expected to give an expression of a possible underlying compartment model.

\subsection{Using Classification to Approximate the Data}

An alternative method is to enforce sparsity by using machine learning techniques. In this method we exploit basis functions that are extracted from data from four healthy patients after manual segmentation of the image into four parts (i) injection site, (ii) blood and surrounding tissue, (iii) kidney and (iv) bladder. For a novel data set we first remove pixels $(x, y)$ for which the time series measurements contain insufficient data to be able to classify reliably. For the remaining pixels an over-segmentation is performed. Each individual segment is then classified into in one of the four classes above. Finally the left and right kidney is automatically separated using a horizontal histogram function of the segmented kidney class. Since the left and right kidney by nature is well-separated it is therefore straightforward to segment them. Prior to the kidney separation a morphological operator is used to obtain a homogenous classification, see Figure 3. The spatial location of the clusters then indicates the possibility of having non-zero weights of the particular bases, these areas are denoted as the support $m_{k}(x, y)$ of compartment $k$. An estimate of $c_{k}(x, y)$ are then obtained from Equation 2. In figure 4 the estimated compartments after classification is displayed.
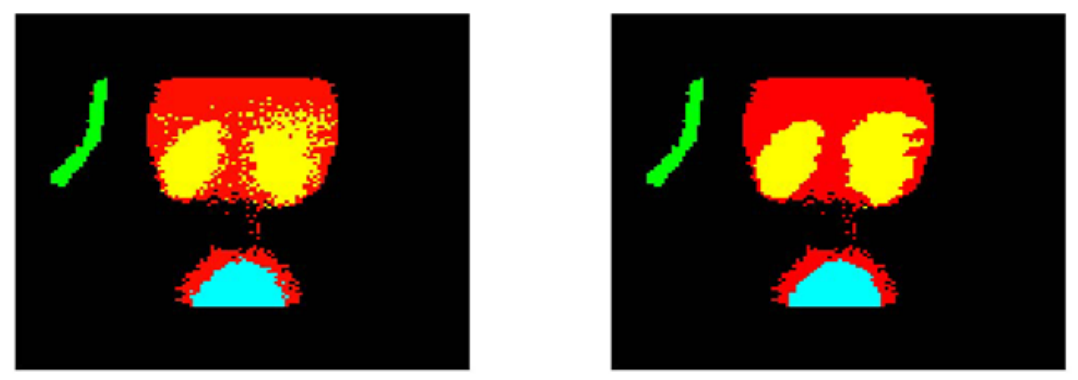

Fig. 3. A figure displaying the classification of pixels into the four classes (left) and the adjusted classification using a morphological operator (right). Legend: Black - pixels not classified due to poor SNR. Green: pixels classified as injection site. Red: pixels classified as blood/tissue. Yellow: pixels classified as kidney. Cyan: pixels classified as bladder. 

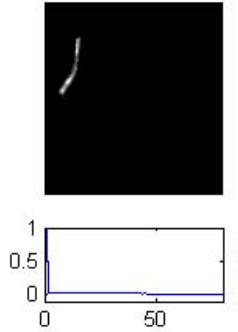
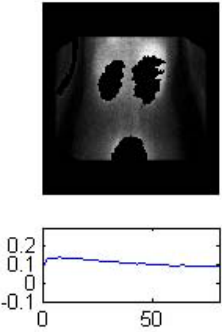
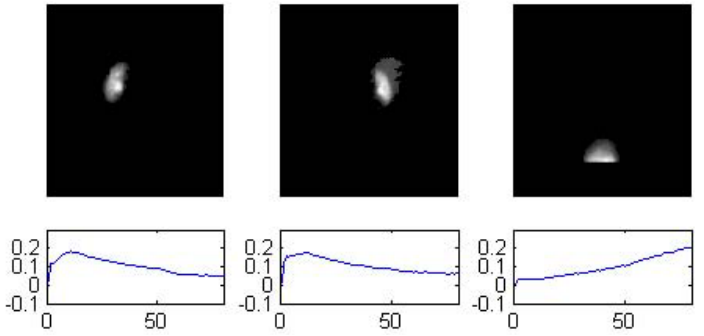

Fig. 4. The compartments generated from classification from left to right (Injection, blood/tissue, left kidney, right kidney, bladder). The lower row holds the bases and the upper row holds the scores. Note the similarity between blood/tissue, left and right kidney TACs, suggesting that the mixture of them has to be estimated.

\section{$2.3 \quad$ Estimation of $b_{k}$}

The composition of each pixel is described by $\mathbf{c}$ and can be used to reduce the influence of one compartment in other compartments and their associated $b_{k}(t)$. The $b_{k}$ s can be estimated using least squares, let $D_{k}(x, y, t)$ denote the data subtracted by the other compartments except for the one to be estimated, $k$, then $b_{k}(t) \mid c_{k}(x, y)$ is estimated as

$$
\widehat{b}_{k}(t)=\min _{b_{k}(t)} \sum_{t=1}^{n_{t}} \sum\left(D_{k}(x, y, t)-b_{k}(t) c_{k}(x, y)\right)^{2} .
$$

\subsection{Estimation of $c_{k}$ Using Positivity Constraints and Spatial Smoothness Priors}

Once estimates of $b_{k}(t)$ and $m_{k}(x, y)$ are given for compartment $k$ it is possible to estimate $c_{k}(x, y)$ using positivity constraints and spatial smoothness priors. If no spatial smoothing is applied, estimation of $c_{k}(x, y)$ becomes independent for each pixel $(x, y)$. If such, the estimation problem becomes $n_{x} n_{y}$ non-negative least squares problems,

$$
\min _{c_{k}(x, y)} \sum_{t=1}^{n_{t}}\left(D(x, y, t)-\sum_{(x, y) \in m_{k}(x, y)} b_{k}(t) c_{k}(x, y)\right)^{2},
$$

of size $n_{t} n_{c}$, where $n_{x}$ and $n_{y}$ are $128, n_{t}$ for our data is 80 and $n_{c}$ is the number of relevant compartments acting on the pixel, in our experiments 1 or 2 . To decrease the computational demand two methods of introducing spatial regularization is considered in this paper. The first is based on linear parametrization of $c_{k}$ so as to enforce spatial smoothness and the other is based on penalizing the second derivatives of $c_{k}$. 
For the linear parametrization, each weight $c_{k}(x, y)$ is parameterized linearly with much fewer parameters or control points. This makes the minimization problem for different pixels connected and the independent pixel problem can instead be written as a large nonlinear least squares problem of the form $d=M c$, where $d$ is a vector of length $n_{x} n_{y} n_{t}$ (for our data 1310720) and $M$ is a sparse matrix of size $n_{x} n_{y} n_{t} \times n_{x} n_{y} K,(1310720 \times 81920)$, where $K$ denotes the number of compartments. The parametrization of $c_{k}$ can now be seen as a linear mapping $c_{k}=R x$, where $x$ denotes the control points. $x$ is then estimated by solving $d=M R x$ in a non-negative least squares manner. This problem is efficiently solved by sparse numerical linear algebra routines since the relevant Fischer matrix $R^{T} M^{T} M R$ is relatively small and has sparse band-diagonal structures.

The first regularization implies smoothing in the neighborhood around the control points. However, the estimation problem is still independent between control points, the second way of regularization connects the control points to enforce spatial smoothing between these as well. To penalize the second derivatives the laplacian of the weights $c_{k}$ is calculated and added into the estimation problem with a proportionality constant $C>0$, i.e. we wish to minimize $|M R x-d|^{2}+C|L x|^{2}$, where the parameter $C$ controls the amount of global regularization applied. The higher $C$ is the smoother the $c_{k}$ s get. In our experiments the selection of $C$ was obtained by manual determination about whether the computed $c_{k}$ s reflected the actual compartment well or not e.g. it is expected that the blood/tissue distribution covers the whole body and have high weights where there is a high concentration of blood.

\subsection{Compartment Modelling}

Given the estimated bases and weights for each compartment and prior knowledge of the interaction between them, the compartment modelling can be performed with the deconvolution analysis approach in mind. With the assumption that the interactions between compartments is linear they can be characterized with an impulse response between the systems' input and output i.e $b_{j}(t)=\left(h * b_{k}\right)(t)$. In matrix notation this can be formulated as $B_{j}=H B_{k}$ where $B_{j}$ is the complete time series of $b_{j}(t)\left(n_{j}=80\right), B_{k}$ is $b_{k}(t)$ 's $\left(n_{k}=n_{j}\right)$ ditto and $H$ is a circulant matrix $\left(H \in \mathbb{R}_{n_{k} \times n_{k}}\right)$. By rearranging the right hand side $H B_{k}=X h$, where $X$ is a left triangular matrix containing elements from $B_{k}$ and $h$ is the impulse response function, $h$ can now be estimated in non-negative least squares sense. To reduce the computational effort regularization using control points is used, similar to the first type introduced in section 2.4, to estimate smooth impulse response functions.

\subsection{Incorporating All Constraint Modalities}

The algorithm of the complete system, incorporating all constraint modalities, can be outlined in two steps - First classification of each pixel is performed to obtain preliminary bases and their support in the image domain. Then an iterative scheme for estimation of $\mathbf{b}$ and $\mathbf{c}$ is performed according to the description in 
Section 2.3, 2.4 and 2.5. The output now consists of the estimated compartments and a compartment model describing their interaction.

\section{Experimental Validation}

In this section the experimental results, validation and a presentation of new features are presented. At first, the experimental results addresses the problem of sparsity, positivity and residual patterns for our data set. The proposed method for $c_{k}$ estimation is also compared to thin plate splines interpolation [14]. Secondly, we use the extracted information from our algorithm to generate the renogram and the parameters computed by the software currently used at SUS and compare the results of the two systems on two healthy patients. At last, a review of possible features using our algorithm is presented.

As mentioned earlier the goal was to come up with an algorithm providing reasonable estimates which fulfills the constraints on positivity, sparseness and spatial smoothness in comparison with the optimal case found by SVD, the SVD results are found in Figure 2. With the introduction of machine learning techniques, the dense $c_{k}$ matrix is reduced to a sparse equivalent containing
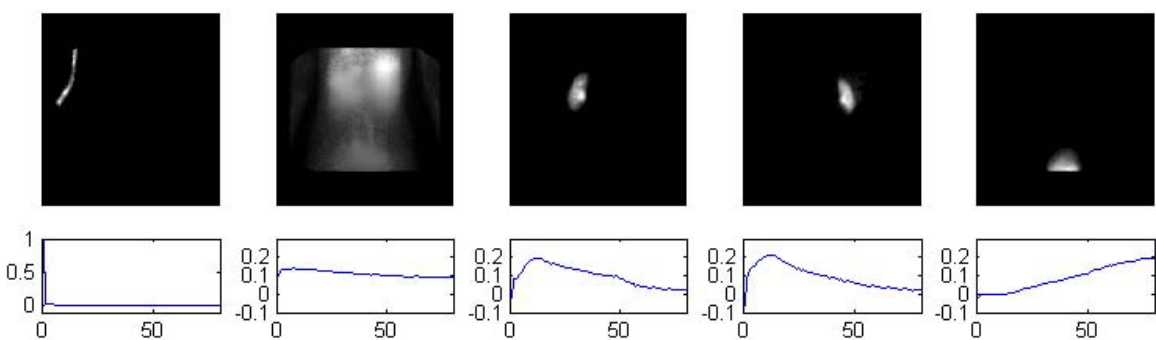

Fig. 5. The injection, blood/tissue, left kidney, right kidney and bladder compartments after thin plate splines interpolation. The lower row holds the bases and the upper row holds the scores.
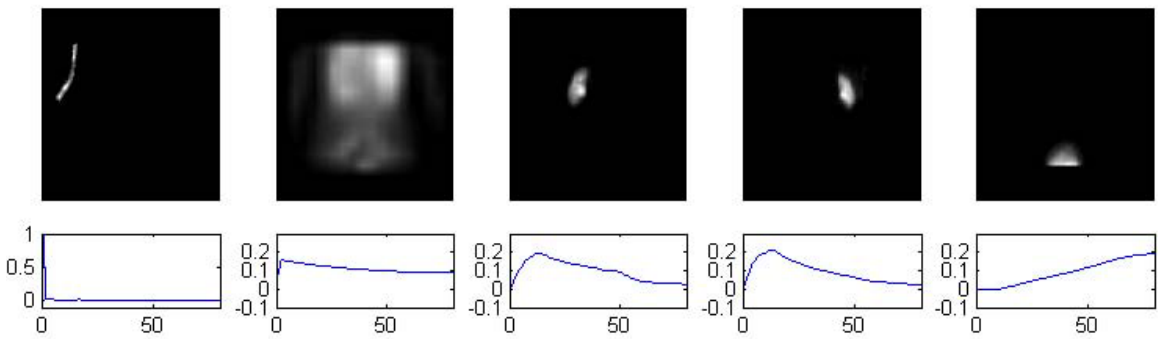

Fig. 6. The injection, blood/tissue, left kidney, right kidney and bladder compartments after the iterative scheme proposed in 2.6. The lower row holds the bases and the upper row holds the scores. 
Table 1. A summary of the results of the algorithm. The system tends to produce sparse weights containing only positive elements and bases also containing only positive elements. The residuals drop a bit as the iteration goes on.

\begin{tabular}{|l|r|r|r|r|}
\hline Step \# & $\% c_{k}(x, y) \neq 0$ & $\% c_{k}(x, y)>0$ & $\% b_{k}(t)>0$ & Residual error \\
\hline \hline PCA & 100 & 63.87 & 57.50 & 27.61 \\
Classification & 20.00 & 100.00 & 100.00 & 34.26 \\
TPS & 23.14 & 100.00 & 95.75 & 33.71 \\
Iteration \#1 & 24.31 & 100.00 & 100.00 & 36.86 \\
Iteration \#2 & 24.33 & 100.00 & 100.00 & 36.23 \\
Iteration \#3 & 24.34 & 100.00 & 100.00 & 36.19 \\
\hline
\end{tabular}

Table 2. A comparison between our system (left) and the current one used at SUS (right). In the renograms (top left and top right) it is seen that our system is able to extract the same shape of the curve.
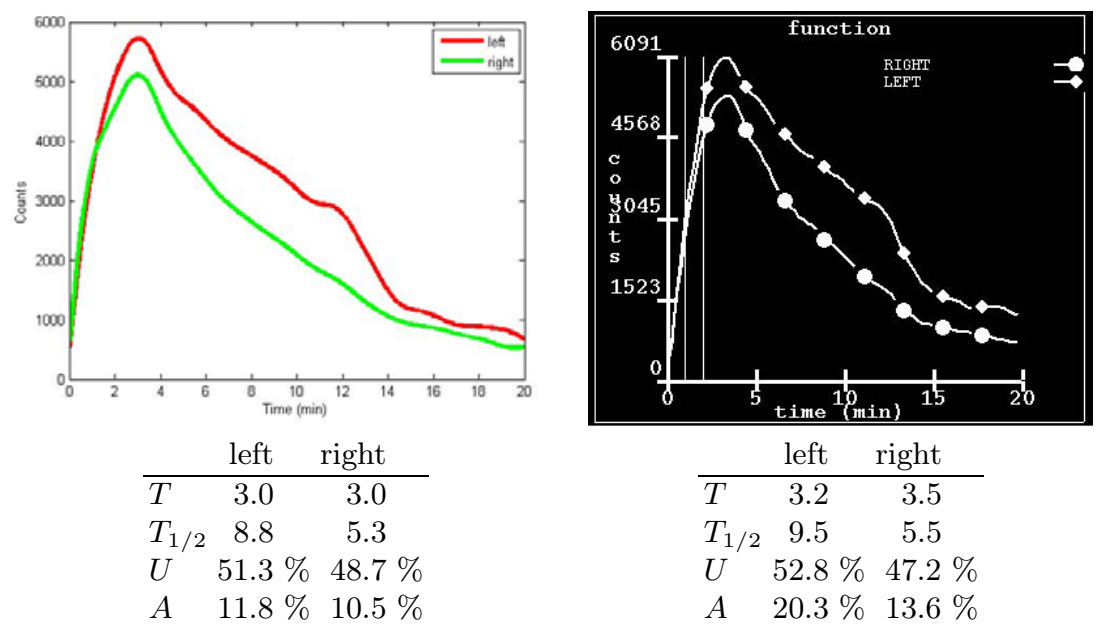

only a fifth of the original non-zero elements, it also produces weights that are non-negative, see Figure 4. The bases are all positive but holds a mixture of information from other compartments as well e.g. the kidney bases are strongly influenced by over- and underlying tissue.

By estimating the influence of other compartments in one compartment a new estimate of $c_{k}$ and $b_{k}$ is obtained. On our data, the thin plate spline interpolation should provide an estimate of tissue in the injection site, kidneys and bladder, see Figure 5 A possible risk by doing this is that to much tissue correction can produce artifacts such as negative elements in $b_{k}$. The interpolation also adds elements to $c_{k}$, which results in denser $c_{k}$ s. With the introduction of positivity and spatial smoothness constraints and three iterations of weights and basis estimation, the $b_{k} \mathrm{~s}$ have converged to bases that have been reduced from influence from other compartments, see Figure 6. The $c_{k}$ is still sparse and the non-zero elements are positive as well. A summary of the results is obtained in Table 1. 

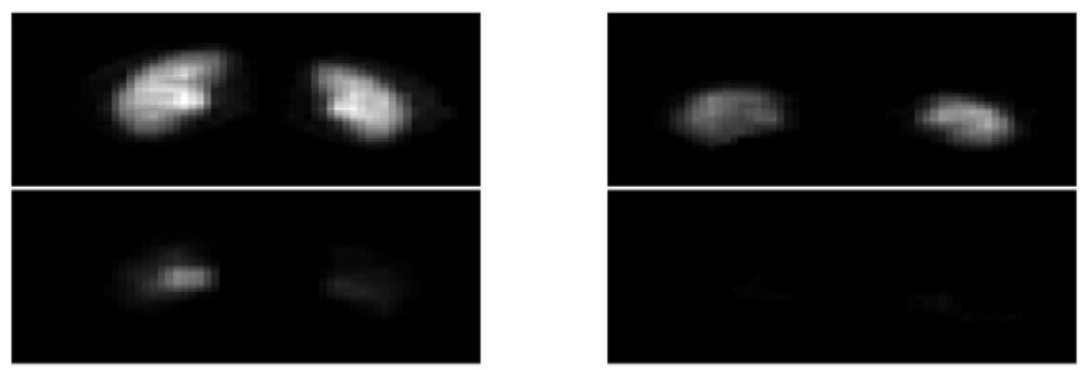

Fig. 7. The coordinate images of the kidney compartment (upper) and bladder compartment (lower) of two healthy subjects (left respective right). There should not be any tracer accumulation if the kidneys are normal, as can be seen in the subject on the right hand side. The left subject's left pelvis accumulates tracer.

A verification study was performed against a program using the manual approach to obtain the renogram. The parameters to test are those currently measured at SUS; Time in minutes to peak of the renogram $T$, time in minutes to reach half peak activity $T_{1 / 2}$, relative uptake by the two kidneys $U$ and residue activity at the end of study $A$. It can be seen in Table 2 that our system is able to reproduce similar results.

By introducing the concept of compartments and the estimation of these our system is able to distinguish non-functional regions of the kidney from normal regions in the particular case of tracer accumulation. Since the bladder compartment represents such an accumulation we can estimate the level of accumulation inside the kidney as we estimate the coordinate image $c_{\text {bladder }} \in m_{\text {kidney }}$. In return we also estimate the remaining function in the functional regions. In Figure 7 it can be seen that the left subject's left renal pelvis suffers a bit from accumulation.

\section{Discussion and Conclusions}

The novel system was tested against a reference system at SUS, using the parameters which is currently used at SUS for assessing the renal function. The results from this comparison study show that our system is able to produce similar results as the present software. However, it needs further development in order to handle pathological kidneys. Since the algorithm just decomposes data in to several compartments it is not sure whether the weights coincides with the true compartments e.g. this can be displayed as an erroneous shape of the kidney if there is no outflow of urine. Validation studies using pathological kidneys therefore have to be performed in the future.

The novel system introduces a new feature - the level of tracer accumulation in the kidney. Since the algorithm outlined in the methods section is able to distinguish the mixture of compartments in each pixel, one could estimate e.g. bladder 
characteristics inside the kidney. If the kidneys suffer from tracer accumulation or obstruction the algorithm induce the presence of bladder inside the kidney. If the generated $c_{\text {bladder }} \in m_{\text {kidney }}$ is compared against a normal database it should be possible to find regions that significantly differs from healthy kidneys. Further studies have to be undertaken to determine whether this feature are relevant for medical assessment or not and how it could be implemented in renography.

All in all, the novel system is successful in automatically retrieving the compartments and use this information to evaluate the composition of compartments in each pixel. With this information it is possible to evaluate characteristics and produce similar results as the present software. Practically, an automated software based on this approach would extract more information and provide better background subtraction since the composition of compartments can be determined. Meanwhile, it reduces the time spent on outlining the different compartments.

The sparseness introduced in this paper is introduced to achieve a compact representation of data. With this representation, spatial smoothness priors can be used in the estimation of the compartments. The implication is that the computational complexity of the method is reduced due to the fact that less pixels is involved in the proposed nonlinear least squares problem.

Recall that the residuals of the least squares estimate should be independent and identically distributed for it to be a valid estimator. However, typical estimates are assumed to attain real numbers whereas counting processes only generate integers. A loss function that penalizes the likelihood for the estimate to come from a counting process could then be considered. However, in our works we keep the estimation with subject to the $L^{2}$-norm.

\section{References}

1. Veltri, P., Vecchio, A., Carbone, V.: Proper orthogonal decomposition analysis of spatio-temporal behavior of renal scintigraphies. Physica Medica 26, 57-70 (2010)

2. Piepsz, A., Sixt, R., Gordon, I.: Performing renography in children with antenatally detected pelvi-ureteric junction stenosis: errors, pitfalls, controversies. The Quarterly Journal of Nuclear Medicine and Molecular Imaging (August 2010)

3. Lawson, R.: Renogram Processing - The Manchester Method. In: XIV. International Symposium on Radionuclides in Nephrourology, Mikulov, Czech Republic, May 11-14 (2010)

4. Hermes Medical Solutions, http://www.hermesmedical.com/index.lasso?id=128

5. Prigent, A., Cosgriff, P., Gates, G.F., Granerus, G., Fine, E.J., Itoh, K., Peters, M., Piepsz, A., Rehling, M., Rutland, M., Taylor Jr, A.: Consensus Report on Quality Control of Quantitative Measurements of Renal Function Obtained From the Renogram: International Consensus Committee From the Scientific committee of Radionuclides in Nephrourology. Seminars in Nucl. Med. 29, 146-159 (1999)

6. Lawson, R.: Quantitative Methods in Renography. In: XIV. International Symposium on Radionuclides in Nephrourology, Mikulov, Czech Republic, May 11-14 (2010)

7. Rutland, M.D.: A comprehensive analysis of renal DTPA studies. Theory and normal values. Nuc. Med. Comm. 6, 11-20 (1985) 
8. Durand, E., Blaufox, M.D., Britton, K.E., Carlsen, O., Cosgriff, P., Fine, E., Fleming, J., Nimmon, C., Piepsz, A., Prigent, A., Samal, M.: International Scientific Committee of Radionuclides in Nephrourology (ISCORN) Consensus on Renal Transit Time Measurement. Semin. Nucl. Med. 38, 82-102 (2008)

9. Moonen, M.: Gamma Camera Renography with 99mTc-DTPA; Assessment of Total and Split Renal Function, Gothenburg (1994)

10. Fine, D.R., Lurie, R.E., Candy, G.P.: An anatomical and physiological model of the renal parenchyma - model development and parametric identification. Physiol. Meas. 15, 407-428 (1994)

11. Meng, L.K., Ng, D., Ghista, D.N., Rudolph, H.: Quantitation of Renal Function based on Two-Compartmental Modeling Renal Pelvis. In: Proceedings of the 2005 IEEE Engineering in Medicine and Biology 27th Annual Conference, Shanghai, China, September 1-4 (2005)

12. Coffey, J.P.: Analysis of compartmental models of type 4 nuclear renograms with calculation of flow rate parameters and instantaneous drainage rates. In: BJU International, vol. 92, pp. 85-91 (2003)

13. Golub, G.H., van Loan, C.F.: Matrix Computations. The Johns Hopkins University Press (1996)

14. Bookstein, F.L.: Principal Warps: Thin Plate Splines and the Decomposition of Deformations. IEEE Transactions on Pattern Analysis and Machine Intelligence 11(6) (June 1989)

15. Hastie, T., Tibshirani, R., Friedman, J.: Elements of Statistical Learning. Springer, New York (2008) 